\title{
Morfometría Geométrica y el Estudio de las Formas Biológi- cas: De la Morfología Descriptiva a la Morfología Cuantitativa
}

\author{
Geometric Morphometry and the Biologic Shapes Study: \\ From the Descriptive Morphology to the Quantitative Morphology
}

\author{
"María Viviana Toro Ibacache; *Germán Manriquez Soto \& **Iván Suazo Galdames
}

TORO, I. M. V.; MANRIQUEZ, S. G. \& SUAZO, G. I. Morfometría geométrica y el estudio de las formas biológicas: de la morfología descriptiva a la morfología cuantitativa. Int. J. Morphol., 28(4):977-990, 2010.

RESUMEN: La morfometría es el estudio de la covariación de la forma con factores subyacentes. Su desarrollo en las últimas décadas ha alcanzado áreas de la biología tradicionalmente dedicadas al estudio descriptivo, como las ciencias morfológicas, las que con las nuevas herramientas morfométricas geométricas han logrado no sólo objetivar la evaluación cuantitativa de los cambios morfológicos sino también la evaluación cualitativa a través de la recuperación de la forma en estudio. Esto es posible gracias a la aplicación de técnicas biométricas, instrumentos y programas computacionales que permiten la captura y análisis de datos en forma de matrices de morfocoordenadas que representan la geometría de un espécimen y no se limitan a la obtención de datos lineales de él como medidas de alto o ancho, elementos que carecen de la precisión y la riqueza de los datos geométricos. El análisis morfométrico geométrico consta de tres etapas fundamentales: obtención de los datos primarios, obtención de las variables de la forma, y análisis estadístico. El extenso uso que se le ha dado en los últimos años en áreas afines a las ciencias morfológicas hace necesario el conocimiento de la técnica tanto con fines formativos como para su aplicación a la solución de problemas en los que la morfología juega un rol esencial.

PALABRAS CLAVE: Morfometría geométrica; Análisis de Procusto; Morfología; Biometría.

\section{INTRODUCCIÓN}

En sus comienzos, la morfología se centró en la descripción de la estructura observada: tejidos, células, órganos, dimensiones, formas, relaciones entre ellos, etc. Las comparaciones entre grupos o poblaciones estaban basadas en el análisis de las diferencias de sus dimensiones lineales y la forma era considerada una cualidad de la estructura, la cual, aunque podía ser detalladamente descrita, no podía ser analizada cuantitativamente. Con el avance de las tecnologías y la estadística, la descripción pasó a la cuantificación y adquirió términos de mayor complejidad, donde no sólo se buscaba comparar parámetros entre grupos de estudio y control, sino que además se intentó buscar relaciones entre éstos, de manera de encontrar asociaciones que permitieran explicar las diferencias observadas.

A partir de la década de 1930, estadísticos como Pearson y Fisher desarrollaron métodos de análisis, y luego los especialistas en biometría, con el objetivo de describir patrones de variación morfológicos intra e intergrupales, co- menzaron a utilizar los análisis estadísticos multivariados análisis de componentes principales, de variables canónicas, función discriminante- a grupos de variables cuantitativas, como largo, ancho, alto y ángulos. Este enfoque es actualmente conocido en biometría como "morfometría tradicional" o "morfometría multivariada" (Marcus, 1990; Rohlf \& Marcus, 1993; Adams et al., 2004). Cabe hacer notar de manera paralela, que en ciencias morfológicas así como en otras áreas que utilizan para la investigación el estudio de formas, el término "morfometría" se utiliza de manera más amplia, abarcando estudios basados en el análisis estadístico univariado de datos lineales de estructuras macro y microscópicas (Zuccarelli, 2004; Duarte et al., 2005; Matamala et al., 2006; Suazo et al., 2007; Aravena et al., 2008; Tatlisumak et al., 2008; Wahl et al., 2009).

Desde entonces, en biometría la definición del concepto de "forma" se ha modificado y precisado. David G. Kendall, estadístico británico, propone a fines de 1970, la 
definición fundamental sobre la que se basa la morfometría geométrica (Kendall, 1977): "Forma es toda la información geométrica que resulta de retirar los efectos de la posición, escala y rotación de un objeto". Esta sola frase ya entrega una idea general de lo que fundamentó en sus orígenes a la morfometría geométrica: analizar la forma independientemente de aquellos componentes que no definen la geometría intrínseca del objeto. La definición de Kendall también da una idea de cómo debieran ser los procedimientos matemáticos -fundamentalmente geométricos- para llevar a cabo el análisis de la forma. Y es ahí donde la morfometría tradicional -y con mayor razón los estudios basados en medidas lineales y estadística univariada- nos entrega herramientas limitadas, debido a que el tamaño como dato numérico es la base de sus análisis, no considerando la geometría del objeto. Es frecuente el uso de este tipo de análisis en estudios antropológicos, pero su valor se ha puesto a prueba, por ejemplo en el estudio del valor diagnóstico de las dimensiones lineales del proceso mastoides como indicador de dimorfismo sexual (Suazo et al., 2008), y donde se observa que la probabilidad de clasificar incorrectamente un cráneo femenino, utilizando este indicador, aumenta si constitucionalmente perteneció a una mujer con un tamaño superior al promedio.

El efecto del tamaño en los estudios biométricos ha sido un tema de preocupación tanto en morfometría tradicional como en morfometría geométrica. En morfometría tradicional, y debido a la alta correlación de las variables lineales con el tamaño, múltiples métodos de corrección fueron propuestos con el fin de dejar de lado el efecto del tamaño y así extraer variables capaces de describir la forma "pura" -en inglés «shape», que la diferencia del término «form» referente a la forma "total" del objeto. Tomemos por ejemplo las variables ancho y alto de dos figuras geométricas. Su valor numérico puede ser el mismo, sin embargo, una figura puede ser un triángulo y la otra un cuadrado. Si consideramos la necesidad de estudiar estructuras altamente complejas, como por ejemplo el esqueleto humano, y relacionar la forma de alguna de sus partes con el origen étnico, las variables lineales podrán claramente no reflejar las diferencias entre un grupo de una etnia u otra, cuyos parámetros de estatura y proporciones no son los mismos, aunque presentan características morfológicas localizadas que los hace claramente distinguibles. Es así como ya en el marco de la morfometría tradicional se propusieron métodos para la corrección del tamaño, pero que no fueron del todo satisfactorios debido a la disparidad de sus resultados (Jungers et al., 1995).

Otro gran problema asociado a los datos en morfometría tradicional es que la definición de las variables en muchos casos, no se daba de acuerdo a un criterio de homología sino que se definían como "largo máximo" o "ancho mínimo". Un ejemplo que grafica esta dificultad fue mencionada por Cantín et al. (2009). Estos autores describieron que el ancho máximo de la apertura piriforme en individuos negroides se encontraba en el tercio inferior de esta apertura, mientras que en los individuos caucasoides ésta se encontraba en el tercio medio. Con ello los puntos correspondientes al ancho máximo en estos sujetos no presentan homología biológica, por lo que el índice nasal, utilizado frecuentemente para la determinación de la afinidad racial, utiliza puntos de referencia no homólogos entre los cráneos analizados. El criterio de homología biológica, presente en morfometría geométrica y al que nos referiremos en mayor profundidad más adelante, otorga restricciones en la elección de aquellos puntos que entregan la información respecto a la forma de un organismo, de manera de obtener resultados que permitan realizar inferencias respecto a la misma (Zelditch et al., 2004). Estas restricciones, además, buscan disminuir el efecto del error de medición. Por ejemplo, no es lo mismo utilizar como referencia los puntos anterior y posterior más prominentes de la calvaria que los puntos bregma o lambda, que son estructuras claramente reconocibles y presentes como tal en un grupo de organismos a comparar. Por otra parte, los análisis que se realizan en morfometría tradicional no permiten la recuperación de la forma en estudio, ya que las matrices que contienen la información, basadas en datos unidimensionales, sólo pueden entregar una representación abstracta de la estructura (Rohlf \& Marcus).

\section{Morfometría Geométrica}

La aplicación de la estadística multivariada al estudio de la covariación de la forma con sus factores casuales dio un gran paso con el uso de datos multidimensionales que logran capturar la geometría de la estructura objetivo. Esto significa que la forma del objeto en estudio no se describe en términos de sus dimensiones, sino de la relación espacial entre sus partes. La información relativa a la geometría de los objetos se puede recuperar, lo que permite la visualización de los cambios morfológicos.

Si pensamos en la utilidad de los resultados obtenidos mediante la aplicación de estos análisis, en comparación con aquellos obtenidos mediante morfometría tradicional, podemos por ejemplo describir cómo se modifica la forma de una estructura durante las distintas etapas de la vida, dónde se localizan estos cambios dentro de esta área y cuáles son esos cambios, ya que pueden ser visualizados (Franchi et al., 2001; Bastir et al., 2006; Cobb \& O'Higgins, 2007). La morfometría tradicional por su parte, nos diría si las variables representativas de la forma de la estructura en estudio son o no distintas en las distintas etapas y, si los 
análisis son suficientes, en qué zona específica se encontrarían esas diferencias. Esto también nos presenta una diferencia a la hora de plantear el diseño experimental en el caso del trabajo con estadística univariada, en el que se deben definir variables a priori, mientras que la morfometría geométrica, con su origen en la estadística multivariada de datos geométricos además nos puede señalar a posteriori, tanto estadística como visualmente, qué región o estructura está siendo afectada por el factor en cuestión y cómo está siendo afectada. Por ello, el análisis con morfometría geométrica permite realizar interpretaciones de alto significado biológico, de las diferencias entre individuos, poblaciones, etc.

La geometría de la estructura se puede capturar como contornos o hitos «landmarks», lo que lleva a distintos métodos de análisis pero que básicamente, siguen tres pasos esenciales: 1) obtención de los datos, 2) obtención de la información que describe la forma "pura" o «shape» y 3) análisis exploratorios y confirmatorios de covariación de la forma con factores causales. Estos pasos serán descritos en mayor profundidad más adelante.

\section{Morfometría Geométrica de Contornos}

Este método se planteó inicialmente para aquellas formas carentes o con una cantidad insuficiente de hitos discretos. Se basa en el supuesto que el borde de una figura es homólogo al borde de otra figura con la que se está comparando. Sin embargo, para obtener dicho contorno -como datose deben colectar puntos de dicho contorno cuyo criterio de homología no siempre es tan claro. Los puntos idealmente deben encontrarse equidistantes o bien aumentar su densidad en aquellas zonas donde la curvatura del contorno sufra cambios más bruscos, cuidando de no sobrecargar de coordenadas el contorno a fin de no generar información redundante (Rohlf, 1990). Las etapas posteriores del análisis varían de acuerdo a si se trata de contornos abiertos o cerrados pero, en general, los puntos digitalizados son ajustados con funciones ad-hoc como las harmónicas del análisis de Fourier y finalmente, los coeficientes de las funciones se utilizan como componentes de la forma, los que conducen al análisis confirmatorio utilizando la estadística multivariada estándar (Rohlf, 1990). Algunos ejemplos de su uso se pueden encontrar en los trabajos de Friess \& Baylac (2003) en relación al estudio de la variación de la forma en cráneos deformados artificialmente, y Krapivka et al. (2007), acerca de la variación morfológica del molusco Mytilus chilensis a lo largo de la costa chilena.

Se han encontrado algunos problemas en los análisis basados en el estudio de contornos relativos a diferencias en los resultados al aplicar distintos métodos para un mismo objetivo (Rohlf, 1990; Adams et al.). Sin embargo, el gran desarrollo de los métodos basados en el uso de hitos discretos, han permitido el estudio de formas que clásicamente presentaron un desafío por sus características, con las ventajas que presenta este tipo de análisis respecto a la confiabilidad de los resultados y otros que serán explicados más adelante.

\section{Morfometría Geométrica de Hitos Discretos}

Es el método más utilizado en la actualidad y la manera en que opera es a través de las etapas fundamentales del análisis:

\section{Obtención de los datos}

Los datos utilizados para el análisis corresponden a un conjunto de hitos representativos de una forma. Un hito (o «landmark») corresponde a un punto del espacio que tiene un nombre -como por ejemplo los puntos cefalométricos $\mathrm{u}$ otros definidos por el operador- $\mathrm{y}$ coordenadas cartesianas $(\mathrm{x}, \mathrm{y})$ en formas bidimensionales, $\mathrm{y}(\mathrm{x}, \mathrm{y}, \mathrm{z})$ en tridimensionales, que son las que describen su posición en el espacio (Bookstein, 1991). En Morfometría Geométrica los conjuntos de coordenadas corresponden a los datos primarios que son luego sometidos a análisis. Es por esto que la selección de los hitos en la planificación de estos estudios es una etapa crucial, ya que de ella dependen no sólo la confiabilidad de los resultados estadísticos, sino que también la cantidad de información que puede obtenerse y con ello las implicancias biológicas de las variaciones observadas, lo cual es de particular importancia en morfología funcional. Para la definición de un conjunto de hitos - configuración o mapa de homologías- adecuado la selección de hitos idealmente debe hacerse bajo los siguientes criterios (Bookstein, 1991; Zelditch et al.; Oxnard \& O'Higgins, 2009):

- Homología: en biología en general y morfología en particular, más que los hitos per se importa la forma que los hitos están definiendo. Las estructuras homólogas son aquellas cuyas semejanzas subyacentes son el resultado de haber sido derivadas a partir de una estructura ancestral común (Gilbert, 2003; Oxnard \& O’Higgins). Se requiere además, que bajo esta definición de homología, los hitos sean discretos, es decir, deben ser claramente diferenciables de las estructuras que lo rodean. Estas características permiten así que cualquier interpretación de la variación observada pueda tener sentido biológico.

- Consistencia en la posición relativa: se refiere a que las formas en estudio no deben diferir radicalmente en la posición de los hitos, lo que ocurre en casos donde puede existir superposición, transposición o bien desaparición de hitos, 
lo que finalmente altera por completo la configuración -la configuración se "dobla" donde se los hitos cambian su topología- generando problemas con el álgebra del análisis. Claramente estos fenómenos son interesantes desde el punto de vista biológico, pero hacen que las estructuras no sean apropiadas para ser sometidas a estos análisis, enfocados en los cambios de forma más que de topología.

- Cobertura adecuada de la forma: los hitos deben "recrear" la forma en estudio. Una cantidad insuficiente de hitos puede llevar a la pérdida de información valiosa, mientras que una cantidad exagerada de hitos lleva a resultados estadísticamente poco confiables si el tamaño de la muestra es insuficiente. El tamaño de la muestra empíricamente debiera ser tres (Monteiro et al., 2002) o cuatro veces la cantidad de hitos (Bookstein, 1996).

- Repetibilidad: a fin de evitar un efecto significativo del error del observador, es importante que el hito sea de fácil localización y que esté lo más claramente definido. Si sólo un punto o un subconjunto de puntos cercanos del mapa de homologías tiene un problema relacionado con su repetibilidad los resultados finales estarán afectados por error sesgado, lo cual puede ser peor si pensamos que pueden llevarnos a intentar explicar lo observado en términos de fenómenos biológicos. Así se entiende la importancia de estimar el efecto del error del observador en Morfometría Geométrica. El criterio de repetibilidad se relaciona con el de homología, pero no necesariamente se dan juntos.

- Coplanaridad: no es lo mismo analizar la fotografía de un pez plano como la reineta (Brama australis), donde la mayoría de sus estructuras e hitos anatómicos se encuentran en un plano anteroposterior, que la fotografía en vista lateral de un cráneo cuyos hitos son medianos y paramedianos. El no tener en cuenta el criterio de coplanaridad puede llevar a una interpretación errónea de resultados, dado que pueden existir variaciones importantes de la forma, que no se aprecian en el plano paralelo a la vista fotográfica sino en el plano frontal, por ejemplo. La ausencia de este problema es una de las ventajas que presenta el análisis tridimensional de los datos.

El criterio de homología se encuentra altamente relacionado con las características anatómicas, funcionales y evolutivas de la estructura donde se situará un hito. La tipología de los hitos se basa tanto en la localización definida de los puntos, como en la explicación que pueden entregar respecto a un fenómeno morfológico. De acuerdo a ello, pueden ser (Bookstein, 1991; Dryden \& Mardia, 1998; Zelditch et al.):

- Hitos tipo 1: corresponden a hitos localizados en la inter- sección de tres estructuras, centros de estructuras muy pequeñas, intersecciones de curvas. Ejemplos de estos hitos son zonas de diferenciación tisular como las intersecciones de suturas o el centro de pequeños forámenes. Este tipo de hito permite observar de manera más precisa el efecto de procesos biológicos como el crecimiento, ya que el hito va a cambiar su posición de acuerdo a lo que esté sucediendo en cualquier parte alrededor de él, debido a que siempre va a estar rodeado de tejidos. Es el tipo de hito que debiera predominar al diseñar un mapa de homologías.

- Hitos tipo 2: corresponden a hitos ubicados en curvaturas máximas, donde existe aplicación de fuerzas biomecánicas como las cúspides de los molares o la parte más prominente de una tuberosidad donde se inserta algún músculo. Debido a su ubicación, no existe información respecto al comportamiento del hito en al menos una dirección.

- Hitos tipo 3: corresponden a hitos extremos cuya definición está dada por estructuras distantes. Gran parte de los hitos usados en morfometría tradicional corresponden a este tipo: diámetros, largos máximos y algunos constructos geométricos. Este tipo de hito se asocia comúnmente al tamaño, por lo que la información referente a la forma "pura" del organismo es bastante escasa. Su presencia en un mapa de homologías debe ser bien explicada y considerada en el momento de interpretar los resultados.

- Pseudo-hitos: corresponden a constructos definidos por términos matemáticos y anatómicos, como puntos tangentes a una curva. Dependiendo de las estructuras y relaciones que describen un pseudohito, puede equivaler a un hito tipo 3.

- Semi-hitos: corresponden a puntos localizados en una curva de acuerdo a la posición de otros hitos o estructuras, y que pueden desplazarse levemente en ella (Bookstein, 1997; Dryden \& Mardia). Como los hitos tipo 3 y pseudos-hitos, la información que puede obtenerse de ellos es menor, y debe considerarse como parte de un todo. Se utilizan para el análisis de formas carentes de hitos homólogos. Debido a su creciente uso en los últimos años en diversas áreas, serán tratados más adelante. Ejemplos de los distintos tipos de hitos se observan en la Fig. 1.

En numerosas ocasiones, el material disponible para la realización de estudios no permite que todos los criterios se cumplan a cabalidad. Por ejemplo, es común el uso de radiografías y fotografías de formas naturalmente tridimensionales como material de trabajo (McIntyre \& Mossey, 2004; Manríquez et al., 2006; Kaliontzopoulou et al., 2007), lo que se contrapone con el criterio de coplanaridad. Es estos casos es imprescindible el uso ma- 


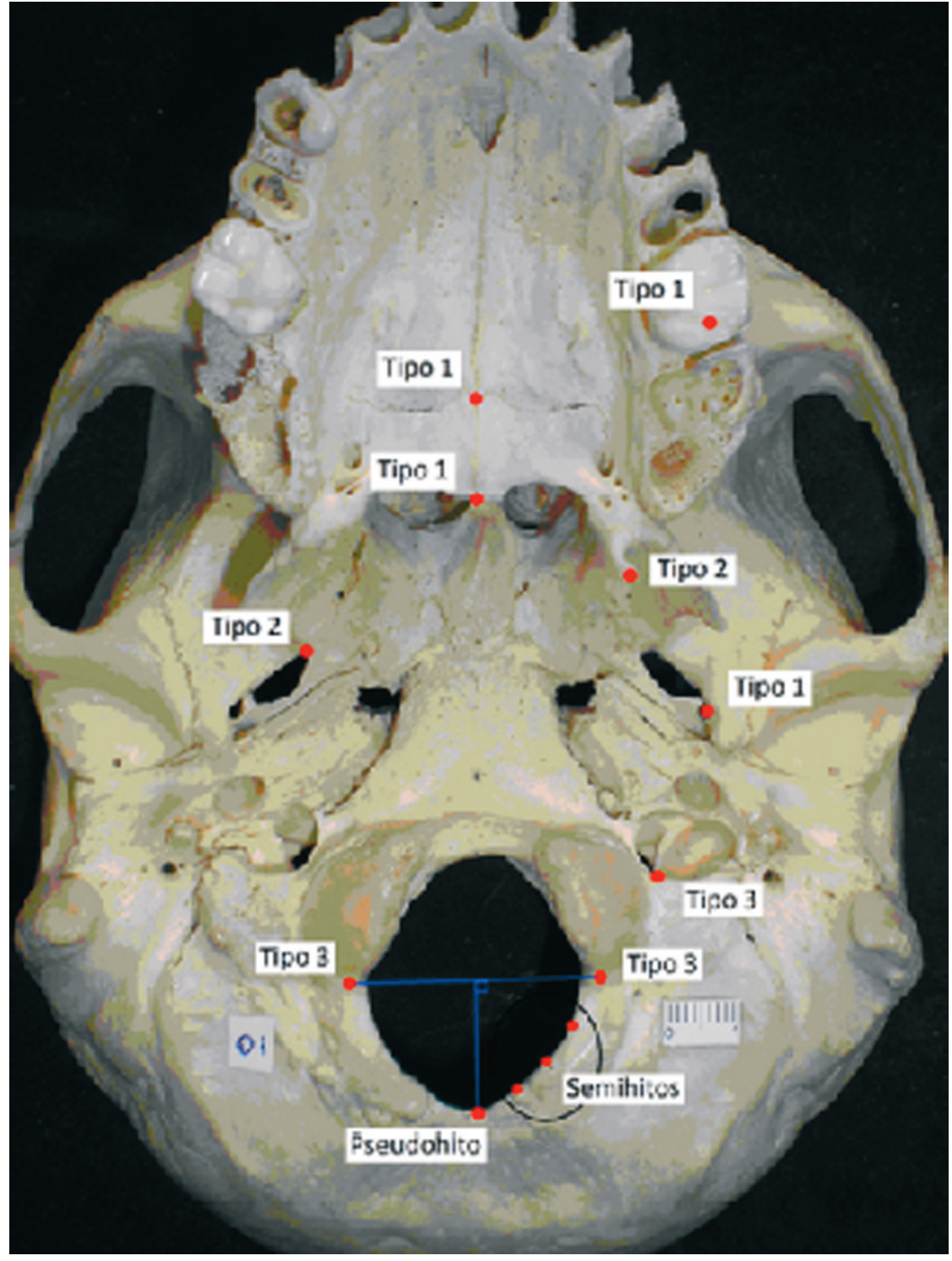

Fig. 1. Imagen externa de la base craneal humana donde se pueden observar ejemplos de los distintos tipos de hitos según Bookstein (1991, 1997). Tipo 1: Fosa de un molar, intersecciones de suturas y centros de formámenes; tipo 2: puntos de máxima curvatura, en forámenes y procesos; tipo 3: hito extremo; pseudohito: constructo en el borde de un foramen definido por dos hitos tipo 3; semihilo: hitos dequidistantes del borde de un foramen entre un hito tipo 3 y un pseudohito.

yoritario de hitos tipo I. En otros casos, la homología puede ser compleja de establecer, la presencia de hitos tipo I es escasa, o bien el material corresponde a fragmentos de material biológico. En éstos, más que descartar el uso de la morfometría geométrica, es importante tener en cuenta estas limitaciones y realizar una interpretación cuidadosa de los resultados. Más aún, el desarrollo de la técnica ha permitido sortear algunos de estos inconvenientes mediante métodos, como la utilización de semihitos en formas carentes de hitos (Bookstein, 1997), o la reconstrucción virtual de estructuras fragmentadas (Benazzi et al., 2009). La Morfometría Geométrica en ningún caso debe ser una restricción para el estudio, sino que busca facilitar la obtención de resultados confiables que lleven a la res- puesta de los problemas planteados. Para una discusión más extensa respecto de la importancia del diseño del mapa de homologías respecto a las preguntas plantadas y la selección de hitos de acuerdo a los criterios elementales, revisar Oxnard \& O’Higgins.

\section{Obtención de la información de la forma "pura" o shape:}

En una segunda etapa, y a fin de obtener la información que describe las formas en estudio, se realizan una serie de procedimientos geométricos y estadísticos basados en la definición misma de la forma. Debemos entonces especificar algunos conceptos:

La teoría de la forma y el análisis morfométrico: Fue ya expuesto que la manera en que Kendall (1977; 1981) define «forma» es lo que sustenta el método en Morfometría Geométrica. La forma de una estructura que será sometida a estudio está definida por su configuración de hitos. Los análisis posteriores no se realizan sobre hitos, sino sobre la configuración completa. Una configuración está formada por $\mathrm{k}$ cantidad de hitos, cada uno de los cuales tiene a su vez $m$ coordenadas: 2 si los datos son bidimensionales y 3 si son tridimensionales. Así, la configuración de hitos se encuentra definida por una matriz de $\mathrm{k}$ líneas por m columnas. A partir de esta configuración, todos los procedimientos que llevan al análisis de la forma libre del efecto de tamaño, rotación y translación se dan en etapas sucesivas y en distintos "espacios morfométricos", constructos geométricos y matriciales que permiten la obtención de los elementos que, finalmente, diferencian una forma de otra: las distancias de Procusto y los componentes de la forma.

Los espacios morfométricos son espacios matemáticos multidimensionales-hiperesferas. Cuando las formas estudiadas son triángulos, 
el espacio que las contiene es una esfera, y en este ejemplo, siendo la esfera una figura conocida, los espacios morfométricos son:

- El espacio de las configuraciones: contiene todas las formas posibles que compartan una configuración $\mathrm{k}$ x m idéntica -en términos de características de la matriz- y que describen un proceso de cambio morfológico estocástico. Este espacio presenta k x m dimensiones (Kendall, 1977; Bookstein; Dryden \& Mardia; Zelditch et al.). Lógicamente, aquellas formas de mayor similitud biológica se encontrarán localizadas en áreas comunes de este espacio. De acuerdo a la definición de Kendall (1977), se deben realizar tres procedimientos, dos de los cuales se llevan a cabo en este espacio: translación y escala, los que definiremos: cada configuración de hitos tiene un centro geométrico o "centroide", que es el punto que describe su posición en este espacio matemático. Si pensamos en dos estructuras cuya única diferencia en su configuración es el centroide, entonces estas formas son idénticas, siempre que sus configuraciones sean centradas, es decir, sus centroides son llevados a un punto común mediante translación. El tamaño en morfometría geométrica -o "tamaño de centroide"corresponde a un concepto geométrico independiente de la forma de la estructura, de manera que dos organismos pueden ser completamente distintos y tener el mismo tamaño de centroide. Equivale a la raíz cuadrada de la suma de las distancias al cuadrado de cada hito desde el centroide (Zelditch et al.). Si usamos como ejemplo un cuadrilátero en el que cada vértice es un hito, y donde se ha unido cada vértice con el centro geométrico mediante las líneas a, b, c y d, su tamaño de centroide o TC está definido por (Fig. 2):

Una vez obtenido el tamaño de centroide, éste puede ser escalado, de manera de llevar varias configuraciones a un tamaño común. Así, después de realizar la translación y escala de las formas, llegamos a un segundo espacio: el de la pre-forma:
- El espacio pre-Kendalliano -pre-shape space-: este espacio tiene menos dimensiones que el de las configuraciones, ya que debido a la translación y la escala, se "pierden" matemáticamente algunas de ellas (para mayor detalle Zelditch et al.). El escalado resta una dimensión, la translación dos en formas planas, y tres en formas con volumen. Sobre este espacio entonces, resta realizar el último procedimiento: la rotación. Ésta genera "áreas" del espacio pre-Kendaliano, en las que se puede rotar una configuración hasta llegar a un punto en que todas las formas se encuentren lo más cerca posible de una forma consenso o promedio -y, por ende, sus diferencias morfológicas estén libres de los efectos ya mencionados. Esta forma consenso tiene una ubicación más centralizada en este espacio preKendalliano. Cuando estas etapas ocurren, se genera un tercer espacio: el espacio de la forma, producto de la rotación translación y escala de las formas. La métrica de los espacios morfométricos es la métrica de Procusto, y su estimador es la distancia de Procusto, que se mide en radianes y captura la curvatura del espacio por descomposición vectorial.

- El espacio de la forma: llamado en Morfometría Geométrica "espacio de Kendall", se genera al eliminar el efecto de la rotación. Este espacio tiene menos dimensiones que el pre-Kendelliano: la rotación en formas planas puede ser sólo sobre un eje, y la de formas con volumen en tres ejes. Recordando que k corresponde al número de coordenadas, y si hacemos los cálculos desde el primer espacio, el espacio de la forma tiene (Zelditch et al.):

En 2D: $2 \mathrm{k}-4$ dimensiones (es decir, $\mathrm{km}-1$ por escala -2 por translación -1 por rotación)

En 3D: $3 \mathrm{k}-7$ dimensiones (es decir, $\mathrm{km}-1$ por escala -3 por translación -3 por rotación)
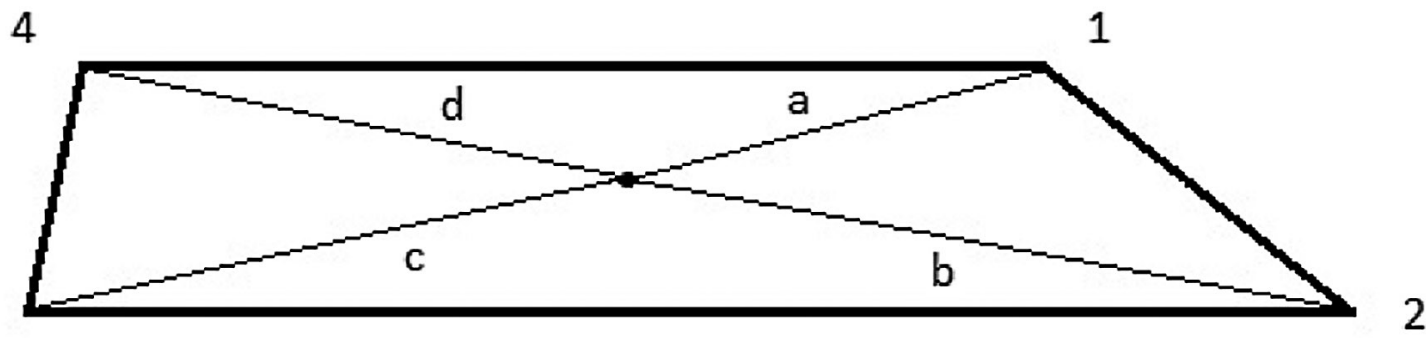

3

$$
T C=\sqrt{a^{2}+b^{2}+c^{2}+d^{2}}
$$

Fig. 2. Método para estimar tamaño de centroide. Para revisar otros métodos, ver Dryden \& Mardia (1998). 
El número de dimensiones se va a relacionar con el número de variables dependientes, o componentes de la forma, que son los datos que van a describir las características de la forma en estudio. Retomaremos este punto más adelante.

En términos generales, lo que hemos relatado corresponde a una minimización de las diferencias de la forma debidas a tamaño, posición y rotación. La manera más común de minimizar las diferencias entre especímenes y respecto de la configuración de consenso corresponde al método generalizado de mínimos cuadrados -GLS, siglas del inglés «General Least Squares»-, más conocido en Morfometría Geométrica como análisis generalizado de Procusto -nombre derivado de la mitología griega (para uso y excepciones ver Zelditch et al.). Este método cumple con la definición de Kendall y en su método de ajuste busca minimizar las distancias de Procusto entre los especímenes dentro del espacio. El efecto sobre los datos originales se muestra en la Fig. 3.

En el espacio de Kendall el tamaño de las configuraciones ahora sufrirá un segundo "tratamiento", ya que el mantener las formas escaladas a un tamaño fijo, como se llevó acabo en los espacios anteriores, hace que las distancias de Procusto -llamadas en este punto "distancias parciales"- sean muy grandes, por lo que se permite en el espacio de la forma un segundo ajuste del tamaño, en el que los tamaños de centroide varían hasta que sus formas encuentren la menor distancia -ahora "distancia completa" de Procusto- de la forma consenso en este espacio matemático. Este enfoque, es el más utilizado en la actualidad, donde el tamaño no es eliminado sino ajustado, e incluso es reutilizado en los análisis subsecuentes, ya que en los últimos años ha habido una tendencia en biología a tener en cuenta esta información, tema que retomaremos más adelante.

a)
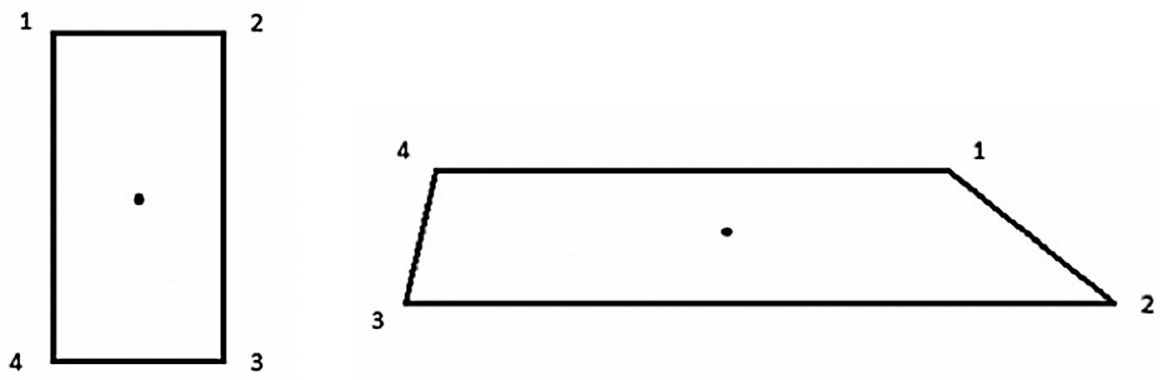

b)

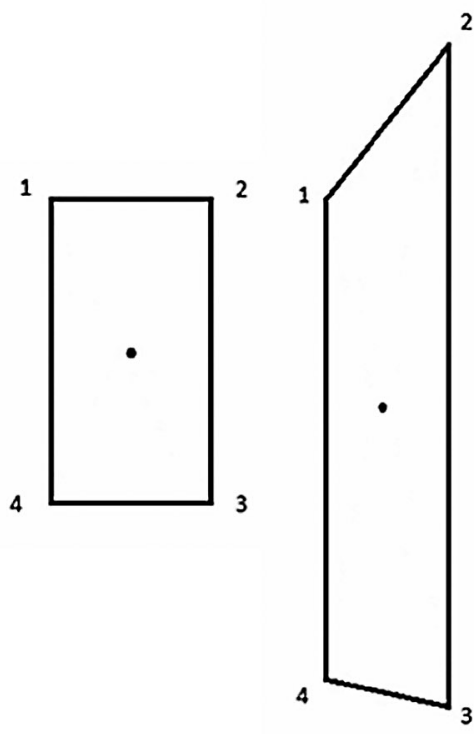

c)

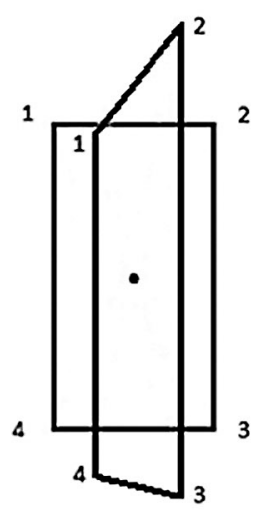

d)

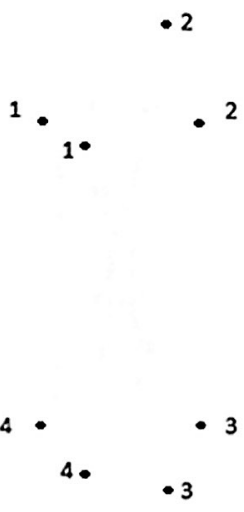

Fig. 3. Minimización de las diferencias de forma de a) dos cuadrláteros a través de b) rotación, c) traslación y escalado a través de la superposición de los centroides. En d) se muestra el resultado de este procedimiento, con las configuraciones de ambos cuadriláteros superpuestas una vez que sus diferencias de posición, rotación y tamaño han sido removidas. 
Si bien la teoría de la forma desarrollada por Kendall es la base del análisis morfométrico geométrico, la estadística multivariada no se aplica a espacios como los descritos anteriormente -que corresponden a espacios matemáticos no Euclidianos o curvos-, por lo que se hace necesario el uso de los "espacios tangentes", en el que las configuraciones son proyectadas de manera estereográfica u ortogonal -lo más utilizado en la actualidad- desde el espacio de Kendall o desde los otros espacios de acuerdo al objetivo del estudio, obteniendo una aproximación bastante cercana a la realidad matemática dentro de dicho espacio. El punto de tan- gencia entre ambos espacios más usado es el de la configuración consenso (Fig. 4).

Con la nueva información de la forma obtenida desde el espacio tangente, ya se pueden realizar diversos análisis donde es posible aplicar métodos estadísticos exploratorios tradicionales como análisis de componentes principales o su análogo análisis de deformación relativa -«relative warps analysis»-, y métodos confirmatorios, temas que veremos más adelante. La figura 4 resume los pasos del análisis morfométrico geométrico.

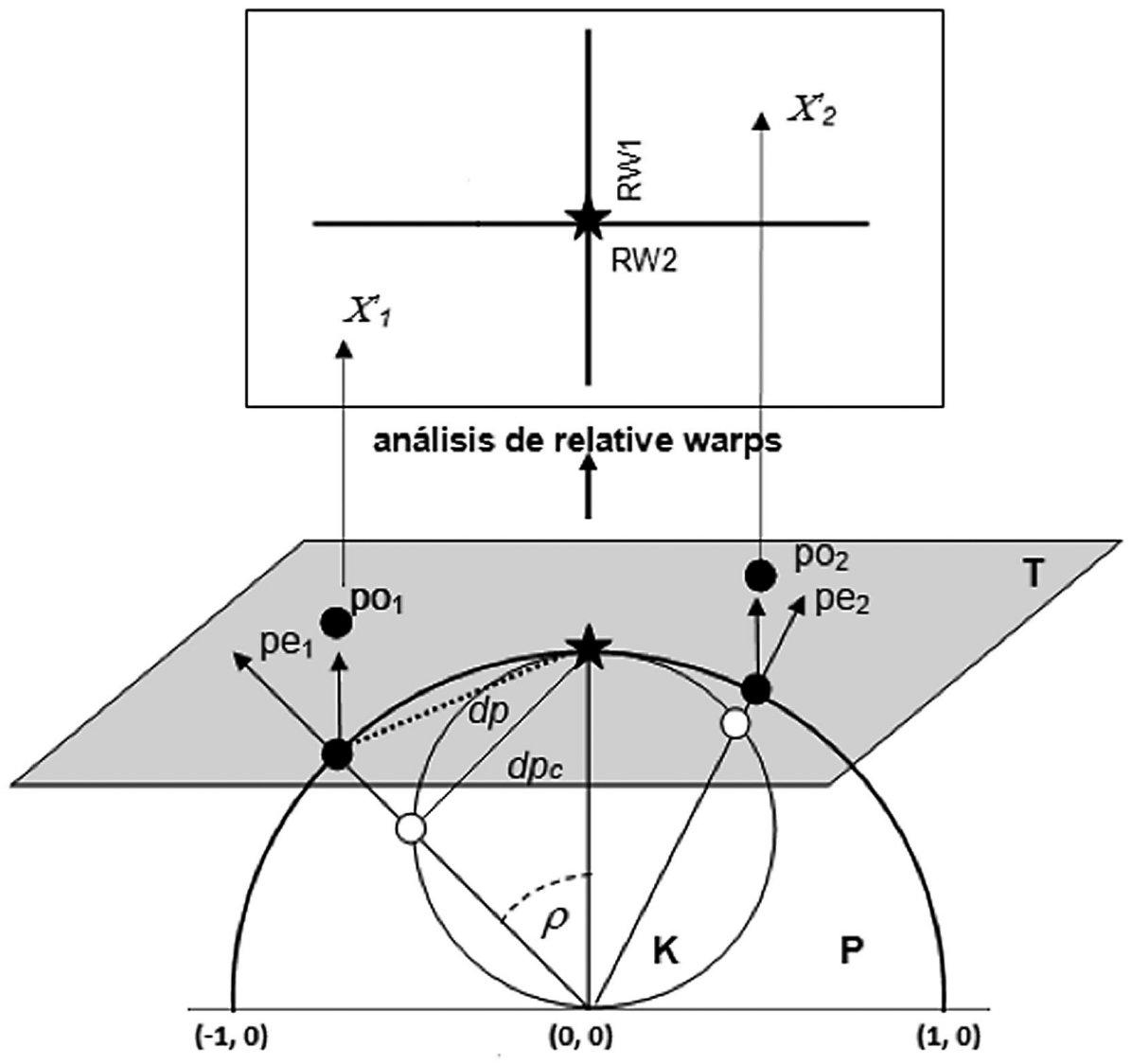

análisis de Procusto

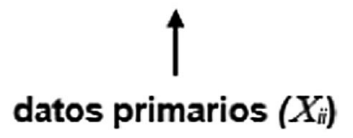

Fig. 4. Esquema que resume los pasos del análisis de morfometría geométrica desde la obtención de datos primarios o matrices de morfocoordenadas X, los cuales son sometidos a análisis de Procusto. El espacio de la pre-forma P y de la forma -de Kendall- K contienen las matrices de datos luego de la eliminación de las diferencias debido a translación, escala y rotación -círculos blancos y negros-, diferencias que en estos espacios son medidos en distancias de Procusto $p$, distancia parcial de Procusto $d p$ y distancia completa de Procusto $d p c$. En el espacio euclidiano T tangente a los espacios $\mathrm{P}$ y K en el punto la configuración consenso $\star$, las configuraciones son proyectadas de manera estereográfica pe $\mathrm{u}$ ortogonal po. Estas últimas $-\mathrm{X}_{\mathrm{n}}{ }_{\mathrm{n}}$ - entonces ocupan distinta ubicación en este plano tangente, lo que es reflejo de sus diferencias morfológicas, que pueden ser cuantificadas por métodos estadísticos multivariados. 
También se puede utilizar una función para obtener variables de la forma, que a la vez permite la visualización de las formas en estudio y sus cambios -una muy reconocida ventaja de la Morfometría Geométrica, como mencionamos anteriormente-, y que es muy utilizada en la actualidad: la función de placa delgada.

Visualización de los cambios morfológicos, obtención de variables dependientes y función de placa delgada:

Una manera de tener una idea global de los cambios de la forma es a través de la función de placa delgada -TPS siglas del inglés «thin plate spline»-, que corresponde a una función de interpolación que permite representar cambios entre una forma y otra como deformación continua. Esta función fue desarrollada por Bookstein (1991), y se basa en la metáfora de una placa de metal infinitamente delgada donde se encuentra localizada una configuración de hitos. Según Bookstein (1991), si se desea transformar la forma definida por esta configuración en otra, habría que sobreponer los hitos homólogos de ambas placas, manteniendo una fija. Como resultado, la placa "blanco" se deforma, lo que ocurrirá en mayor medida si las formas comparadas son muy distintas. Esta deformación se mide en términos de "energía de torsión", la cual se puede medir, constituyendo variables de forma que abarcan todas las dimensiones del espacio de la forma - 2k-4 para formas planas y $3 \mathrm{k}-7$ para formas con volumen. La función TPS es compatible con la superimposición de Procusto, y las variables de la forma obtenidas -«partial warps»-, pueden ser sometidas a análisis estadísticos convencionales. La función TPS es la más utilizada en la actualidad para la realización del análisis morfométrico (ver ejemplos de excepciones en Bookstein, 1991; Zelditch et al.), y está presente en los programas computacionales más usados actualmente.
La manera en que la placa se deforma puede visualizarse de dos maneras: utilizando una grilla en su superficie -herramienta basada en el trabajo de Thompson (1917)-, y si la deformación conlleva a una expansión o contracción, o cualquier otro resultado en el que las líneas de la grilla mantengan su paralelismo, estamos en presencia de deformación uniforme -o afín-, mientras que si la deformación ocurre en zonas localizadas de la forma en estudio, las líneas pierden su paralelismo, lo que se denomina deformación no uniforme -o no afín- (Fig. 5). Las formas biológicas normalmente presentan ambos tipos de deformación.

Tanto la deformación uniforme como la no uniforme pueden traducirse en términos numéricos, y ambas en conjunto definen los cambios morfológicos de una estructura respecto a otra, generalmente la figura consenso. Algunos programas, como los de la serie TPS (Rohlf, 2003; 2008) otorgan la posibilidad de realizar análisis diferenciados de los dos componentes. Los números que describen los cambios morfológicos corresponden a los partial «warps scores». Los «partial warps» son los valores principales de la matriz de energía de torsión, por lo que los «partial warps» scores son valores expresados en los ejes X e Y. Hay tantos «partial warps scores» como dimensiones tiene el espacio de Kendall de la muestra en estudio, y de ellos, algunos describen la deformación uniforme -los que se conocen como los «partial warps $0 »$, por lo que se cuentan aparte- y el resto la no uniforme. Los valores que representan la deformación uniforme están ordenados de menor a mayor, representando desde la más bajo a la mayor cantidad de energía de torsión (Bookstein, 1991; Rohlf, 1999; Zelditch et al.). Los partial «warps scores» pueden ser utilizados como variables dependientes en análisis estadísticos multivariados clásicos.

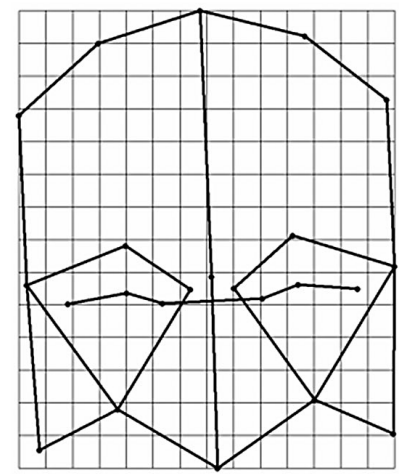

Configuración consenso

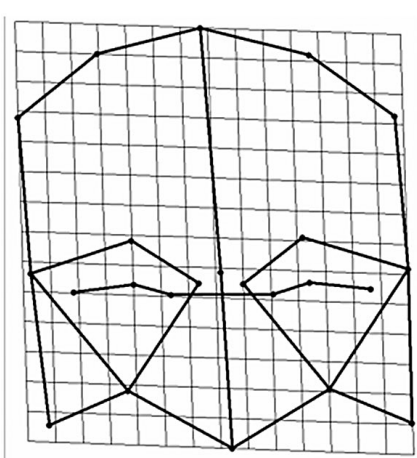

Deformación uniforme

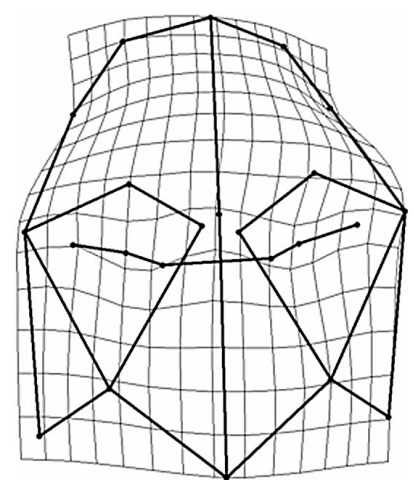

Deformación no uniforme

Fig. 5. Ejemplos de los distintos tipos de deformación utilizando las grillas basadas en el trabajo de Thompson (1917) en una configuración de 23 hitos representativos de la anatomía craneal humana en el plano frontal. Se muestra la manera en que la grilla mantiene el paralelismo de sus líneas en la deformación uniforme, lo que no ocurre con la deformación no uniforme. 


\section{Análisis exploratorios y confirmatorios de covariación de la forma y factores causales.}

Con la proyección del espacio de Kendall a un espacio tangente la información que describe la forma en estudio, puede ser estudiada de diversas maneras, tales como análisis exploratorios, análisis de componentes principales -ACP- (O`Higgins, 2000; Zelditch et al.; Slice, 2007), análisis de variantes canónicas -AVC- (Zelditch et al.), análisis de deformación relativa -«relative warps analysis», que corresponde al ACP de los «partial warps scores»- (Rohlf, 1999; Zelditch et al.), y otros análisis para la puesta a prueba de hipótesis, como análisis multivariado de la varianza MANDEVA-, regresiones multivariadas (Zelditch et al.) y varios test no paramétricos. Así, las morfocoordenadas permiten probar la diferencias entre formas promedio, probar el grado de correlación y covariación de la forma con el tamaño, covariación de la forma con factores externos, la covariación entre formas distintas a través del análisis parcial de cuadrados mínimos -«partial least squares analysis»(Rohlf \& Corti, 2000), el estudio de la variación de formas simétricas y pruebas de la presencia de los distintos tipos de asimetría (Klingenberg \& McIntyre, 1998; Klingenberg et al., 2002). Estas más frecuentes aplicaciones y otras presentan claros aspectos de interés en las ciencias morfológicas, y están disponibles en gran parte de los programas destinados a Morfometría Geométrica y que, además, se encuentran disponibles de manera gratuita, como Morphologika (O’Higgins \& Jones, 2006), MorphoJ (Klingenberg, 2008), tpsRegr (Rohlf, 2003), tps Relative Warps (Rohlf, 2008), y otros programas más antiguos o pagados. Estos programas además ofrecen la posibilidad de visualización de los cambios morfológicos entre los especímenes de la muestra de acuerdo a los resultados del análisis (ejemplo en Fig. 6), lo que desde el punto de vista biológico es una gran ventaja.

\section{Morfometría Geométrica de Hitos Discretos y el Estu- dio de las Formas que Carecen de Hitos Anatómicos Homólogos.}

Los avances logrados en el campo de la Morfometría Geométrica de hitos discretos no tendrían utilidad en el análisis de formas que carecen de hitos anatómicos homólogos, como las líneas o superficies curvas. La necesidad de situar puntos en estas estructuras, de manera que puedan ser tratadas como hitos en un análisis tipo, llevó al desarrollo de los semihitos (Bookstein, 1997), hitos que son localizados por el operador de acuerdo a distintos criterios, siendo uno de los más utilizados los intervalos aproximadamente equidistantes entre dos hitos (para otros métodos de localización, ver Zelditch et al.), y que caen en la categoría de hitos geométricos, ya que por definición no siguen criterios de homología biológica, sino que más bien es el contorno de la forma estudiada el que es homólogo. Debido a este criterio, su aporte en el estudio de formas biológicas es distinto al de los hitos tipos 1 y 2 .

Para que los semihitos sean "tratados" como hitos, deben, en primer lugar desplazarse a lo largo de la línea o superficie curva de tal manera que las diferencias en la localización entre los especímenes sean minimizadas, ya sea en términos de distancias de Procusto o energía de torsión. Así, el resultado corresponde a las diferencias observadas en la curva como un todo y no en zonas particulares de ella.

El uso de semihitos no se ha masificado aún, ya sea por motivos de desconocimiento de su uso o porque existen algunos reparos debido a la disparidad de resultados según los distintos enfoques y los aún escasos softwares para trabajar con semihitos (Slice), lo que a su vez refleja las distintas opiniones que los morfometristas tienen del tema, tanto los usuarios como programadores. Se han encontrado diferencias además en la precisión de la localización de hitos geométricos versus hitos biológicos al utilizar los mismos modelos y método de adquisición de datos -digitalizador manual sobre el espécimen físico o digitalización virtual en modelos computacionales- (Sholts et al., 2010), lo que podría presentar problemas en algunos trabajos. Aún así, en ciencias biológicas, diversas áreas han utilizado semihitos en los últimos años en trabajos relativos a la morfología del segundo premolar inferior en homínidos (Martinón-Torres et al., 2006), reconstrucción de cráneos arqueológicos (Gunz et al., 2005; 2009), el estudio de la forma del disco óptico y su relación con el glaucoma (Sanfilippo et al., 2009), relación entre morfología vertebral y madurez esqueletal (Chatzigianni \& Halazonetis, 2009), por mencionar algunos ejemplos.

La Morfometría Geométrica tiene, por lo tanto, una gran cantidad de campos para su desarrollo en ciencias morfológicas. Ejemplos de su aplicación puede ser la evaluación del crecimiento para fines clínicos, y no sólo de estudio antropológico o biológico estricto. Cualquier estructura que se encuentra en desarrollo puede ser susceptible de ser analizada mediante Morfometría Geométrica según sus características. En antropología forense la Morfometría Geométrica presenta gran utilidad. Como ya mencionamos, los indicadores de dimorfismo sexual o afinidad racial más utilizados se basan en morfometría lineal y ya se sabe que presentan limitaciones y una disminución de la exactitud debido a las diferencias de tamaño (Suazo et al., 2009a, 2009b; Zavando et al., 2009a, 2009b), por lo que aquellos indicadores basados en forma presentan un mejor rendimiento (Suazo et al., 2009c). En estos casos, la evaluación cuantitativa de la forma utilizando Morfometría Geométrica resultará útil para la evaluación forense del cráneo (Kimmerle et al., 2008). 

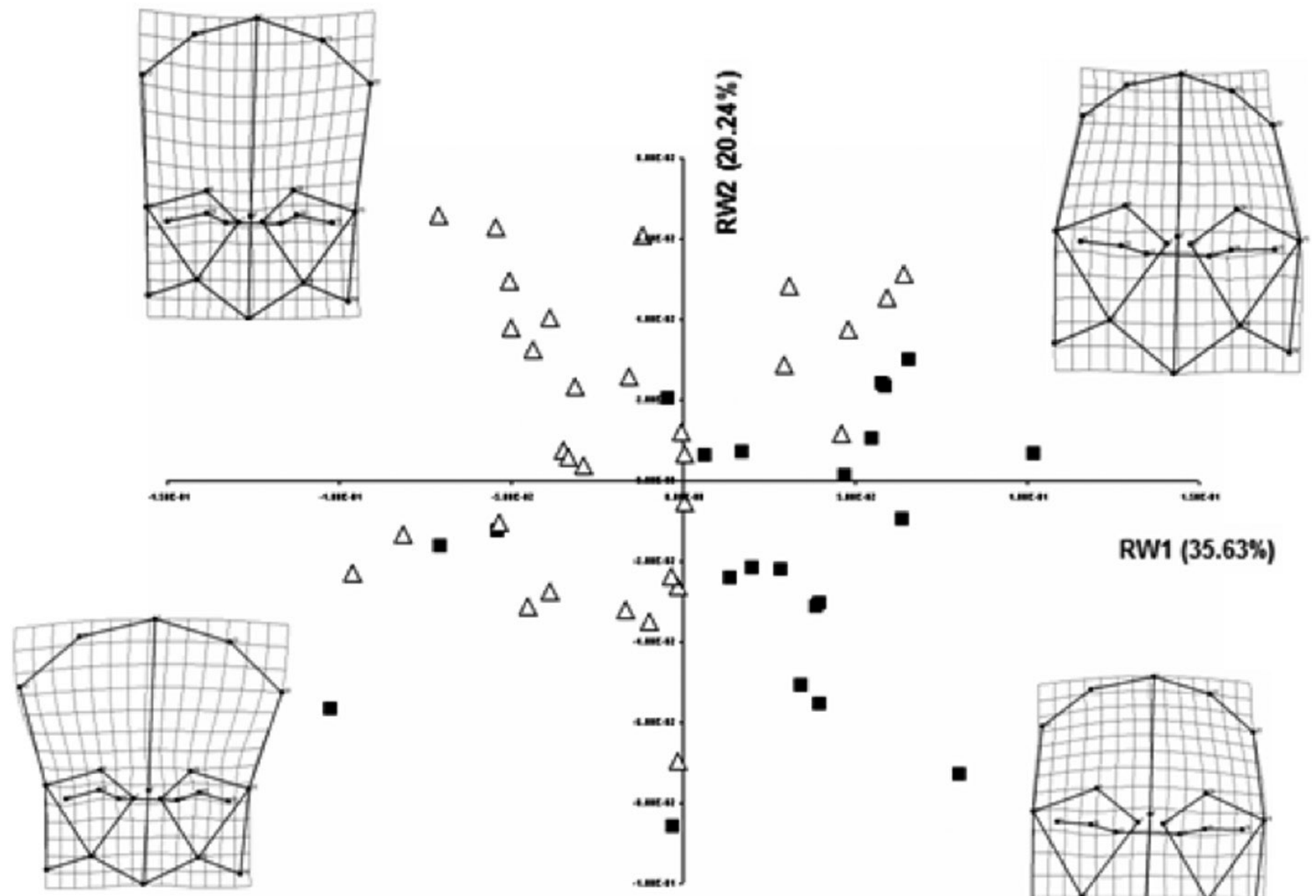

RW1 (35.63\%)

Fig. 6. Ejemplo de un análisis de deformación relativa para la variación morfológica de configuraciones de 23 hitos representativoscráneos no deformados -cuadros negros- y deformados artificialmente -triángulos blancos- (tomado de Toro, 2010). Obsérvese su estructura similar al análisis de componentes principales. Mediante la función de placa delgada, es posible describir las variaciones de la forma según la posición de la configuración en el plano.

Si bien la morfometría geométrica en ciencias morfológicas y afines, como se observa en los ejemplos citados en este trabajo, ha sido más ampliamente utilizada en el análisis de estructuras macroscópicas, particularmente en análisis de cráneos de diversas especies, hay áreas de las ciencias morfológicas de gran potencial en las que hay muy poco o ningún uso de esta técnica. Es el caso de la histología y embriología, en las que, teniendo en cuenta las los aspectos relativos a homología y método en general, la Morfometría Geométrica podría ser un aporte. Por ejemplo, en la determinación de los cambios morfológicos embrionarios utilizando modelos virtuales, o el diagnóstico histopatológico de las formas de anaplasia en células tumorales. Una célula neoplásica que comienza un proceso de desdiferenciación cursa por distintos estadios, los cuales no son discretos, y su clasificación depende de la opinión sub- jetiva del observador quien se basa en criterios cualitativos. La Morfometría Geométrica aplicada al análisis de la forma de las células neoplásicas podría establecer un porcentaje de variación de forma de las células estudiadas, haciendo más preciso el diagnóstico.

En conclusión, la Morfometría Geométrica, gracias a sus características relativas a la naturaleza de los datos primarios y la aplicación de estadística multivariada. es una poderosa herramienta para la evaluación objetiva de las variaciones de forma y que, debido a sus continuos avances, presenta una amplia aplicabilidad en los estudios de las ciencias morfológicas, por lo que su conocimiento no sólo es importante para introducir una nueva técnica de análisis, sino que también lo es como parte del conocimiento general fundamental en la formación y actualización de un morfólogo. 
TORO, I. MV.; MANRIQUEZ, S. G. \& SUAZO, G. I. Geometric morphometrics and the study of biologic shapes: from descriptive to quantitative morphology. Int. J. Morphol., 28(4):977-990, 2010.

SUMMARY: Morphometrics is the study of co-variation of biological form and its causes. Its development over the last decades has reached several biological sciences with a traditional descriptive approach, such as morphological sciences. The new geometric morphometric tools allow not only objective quantitative analysis, but also to assess qualitative traits due to the chance of recovering the form under study. This is possible because of the application of biometry techniques, instruments and software that allow the acquisition and analysis of shape coordinates that represent the geometry of the specimen, and that are not limited to obtaining linear data that lack of precision and amount of information of geometric data. Geometric morphometric analysis consists of three fundamental steps: obtaining primary data, obtaining shape variables and statistical analysis. The extensive use of this technique in areas related to morphological sciences over the last years makes geometric morphometrics a must-know subject for morphologists, in general knowledge, as well as for its use in solving problems where morphology plays an essential role.

KEY WORDS: Geometric morphometrics; Procrustes analysis; Morphology; Biometry.

\section{REFERENCIAS BIBLIOGRÁFICAS}

Adams, D. C.; Rohlf, F. J. \& Slice, D. E. Geometric morphometrics: ten years of progress following the "revolution". Ital. J. Zool., 71:5-16, 2004.

Aravena, T. P.; Suazo, G. I.; Cantín, L. M; Troncoso, P. J. \& Coronado, G. C. Biomethrics study of the upper and lower labial artery in human cadavers. Int. J. Morphol., 26(3):573-6, 2008.

Bastir, M.; Rosas, A. \& O'higgins, P. Craniofacial levels and the morphological maturation of the human skull. $J$. Anat., 209(5):637-54, 2006.

Benazzi, S.; Stansfield, E.; Milani, C. \& Gruppioni, G. Geometric morphometric methods for three-dimensional virtual reconstruction of a fragmented cranium: the case of Angelo Poliziano. Int. J. Legal Med., 123(4):33344, 2009.

Bookstein, F. L. Morphometric tools for landmark data: Geometry and biology. Cambridge, Cambridge University Press, 1991.

Bookstein, F. L. Combining the tools of geometric morphometrics. In: Marcus, L. F.; Corti, M.; Loy, A.; Naylor, G. J. P. \& Slice, D. Advances in morphometrics. New York, Plenum Press, 1996. pp.131-51.

Bookstein, F. L. Landmark methods for forms without landmarks: Morphometrics of group differences in outline shape. Med. Image Anal., 1(3):225-43, 1997.

Cantín, L. M.; Suazo, G. I. C; Zavando, M. D. A \& Smith, R. L. Sexual dimorphism determination by piriform aperture morphometric analysis in Brazilian human skulls. Int. J. Morphol., 27(2): 327-31, 2009.
Chatzigianni, A. \& Halazonetis, D. Geometric morphometric evaluation of cervical vertebrae shape and its relationship to skeletal maturation. Am. J. Orthod. Dentofacial Orthop., 136(4):481, 2009.

Cobb, S. \& O’Higgins, P. The ontogeny of sexual dimorphism in the facial skeleton of the African apes. J. Hum. Evol., 53(2):176-90, 2007.

Dryden, I. L. \& Mardia, K. V. Statistical shape analysis. Chichester, John Wiley and Sons, 1998.

Duarte, A. T.; Barbosa, Jr. A. A. \& Arruda, S. Morphometric analysis of granulomas induced by Mycobacterium bovis suggests an influence of IFN-Gamma on the generation and modulation upon granulomatous inflammatory response in the different tissues. Int. J. Morphol., 23(4):317-22, 2005.

Franchi, L.; Baccetti, T. \& McNamara, J. A. Jr. Thin-plate spline analysis of mandibular growth. Angle Orthod., 71(2):83-9, 2001.

Friess, M. \& Baylac, M. Exploring artificial cranial deformation using elliptic Fourier analysis of Procrustes aligned outlines. Am. J. Phys. Anthropol., 122(1):11-22, 2003.

Gilbert, S. F. Biología del Desarrollo. $7^{\mathrm{a}}$ ed. Buenos Aires, Médica Panamericana, 2003.

Gunz, P.; Mitteroeker, P. \& Bookstein, F. L. Semilandmarks in three dimensions. In: Modern Morphometrics in Physical Anthropology. Slice, D. E. (ed) Chicago, University of Chicago Press, 2005. pp.73-98. 
Gunz, P.; Mitteroecker, P.; Neubauer, S.; Weber, G. W. \& Bookstein, F. L. Principles for the virtual reconstruction of hominin crania. J. Hum. Evol., 57(1):48-62, 2009.

Jungers, W. L.; Falsetti, A. B. \& Wall, C. E. Shape, relative size, and size-adjustments in morphometrics. Yrbk. Phys. Anthropol., 38:137-61, 1995.

Kaliontzopoulou, A.; Carretero, M. A. \& Llorente, G. A. Multivariate and geometric morphometrics in the analysis of sexual dimorphism variation in Podarcis lizards. J. Morphol., 268(2):152-65, 2007.

Kendall, D. G. The diffusion of shape. Adv. Appl. Prob., 9:428-30, 1977.

Kendall, D. G. The statistics of shape. In: Bartnett, V. Interpreting multivariate data. New York, John Wiley \& Sons, 1981. pp.75-80.

Kimmerle, E. H.; Ross, A. \& Slice, D. Sexual dimorphism in America: Geometric morphometric analysis of the craniofacial region. J. Forensic Sci., 53(1):54-7, 2008.

Klingenberg, C. P.; Barluenga, M. \& Meyer, A. Shape analysis of symmetric structures: Quantifying variation among individuals and asymmetry. Evolution; 56(10):1909-20, 2002.

Klingenberg, C. P. \& McIntyre, G. Geometric morphometrics of developmental instability: Analyzing patterns of fluctuating asymmetry with Procrustes methods. Evolution, 52(5):1363-75, 1998.

Klingenberg, C. P. Morpho. J. UK, Faculty of Life Sciences, University of Manchester, 2008.

Krapivka, S.; Toro, J. E. C.; Alcapán, A. C.; Astorga, M.; Presa, P.; Pérez, M. \& Guínez, R. Shell-shape variation along the latitudinal range of the Chilean blue mussel Mytilus chilensis (Hupe 1854). Aquaculture Res., 38:1770-7, 2007.

Manríquez, G.; González-Bergás, F. E.; Salinas, J. C. \& Espoyeus, O. Deformación intencional del cráneo en poblaciones arqueológicas de Arica, Chile: Análisis preliminar de morfometría geométrica con uso de radiografías craneofaciales. Chungará, 38(1):13-34, 2006.

McIntyre, G. T. \& Mossey, P. A. Parental craniofacial morphology in orofacial clefting. Eur. J. Orthod., 26(4):375-84, 2004.
Marcus, L. F. Traditional morphometrics. En: Proceedings of the Michigan Morphometrics Workshop. Rohlf, F. J. \& Bookstein, F. L. (Eds). Special Publication Number 2. Ann Arbor, Michigan, The University of Michigan Museum of Zoology, 1990. pp.77-122.

Martinón-Torres M.; Bastir, M.; Bermúdez de Castro, J. M.; Gómez, A.; Sarmiento, S.; Muela, A. \& Arsuaga, J. L. Hominin coger second premolar morphology: evolutionay inferences through geometric morphometric analysis. J. Hum. Evol., 50(5):523-33, 2006.

Matamala, F.; Fuentes, R. \& Ceballos, M. Morfología y morfometría del disco de la articulación témporomandibular en fetos y adultos humanos. Int. J. Morphol., 24(2):245-50, 2006.

Monteiro, L. R.; Diniz-Filho, J. A.; dos Reis, S. F. \& Araújo, E. D. Geometric estimates of heretability in biological shape. Evolution, 56(3):563-72, 2002.

O'Higgins, P. The study of morphological variation in the hominid fossil record: Biology, landmarks and geometry. J. Anat., 197:103-20, 2000.

O'Higgins, P. \& Jones, N. Tools for statistical shape analysis. Hull York Medical School, 2006. Available in: http:// www.york.ac.uk/res/fme/resources/software.htm

Oxnard, C. \& O’Higgins, P. Biology clearly needs Morphometrics! Does Morphometrics need Biology? Biol. Theory, 4:84-97, 2009.

Rohlf, F. J. Fitting curves to oulines. En: Proceedings of the Michigan Morphometrics Workshop. Rohlf, F. J. \& Bookstein, F. L. (Eds). Special Publication Number 2. Ann Arbor, Michigan, The University of Michigan Museum of Zoology, 1990. pp.167-77.

Rohlf, F. J. Shape statistics: Procrustes superimpositions and tangent spaces. J. Classification, 16:197-223, 1999.

Rohlf, F. tpsRegr, Shape regression, versión 1.28. New York, Department of Ecology and Evolution, State University of New York at Stony Brook, 2003.

Rohlf, F. tps Relative Warps, versión 1.46. New York, Department of Ecology and Evolution, State University of New York at Stony Brook, 2008.

Rohlf, F. J. \& Corti, M. Use of two-block partial least-squares to study covariation in shape. Syst. Biol., 49(4):740-53, 2000 . 
Rohlf, F. J. \& Marcus, L. F. A revolution in morphometrics. Trends in Ecology and Evolution 8(4):129-32, 1993.

Sanfilippo, P. G.; Cardini, A.; Hewitt, A. W.; Crowston, J. G. \& Mackey, D. A. Optic disc morphology - Rethinking shape. Prog. Retin. Eye Res., 28(4):227-48, 2009.

Sholts, S. B.; Flores, L.; Walker, P. L. \& Wärmländer, S. K. T. S. Comparison of coordinate measurement precision of different landmark types on human crania using a 3D laser scanner and a 3D digitiser: Implications for applications of digital morphometrics. Int. J. Osteoarchaeol., DOI:10.1002/oa.1156, 2010.

Slice, D. E. Geometric Morphometrics. Ann. R. Anthr, 36:261-81, 2007.

Suazo, G. I. C.; Morales, H. C. A.; Cantín, L. C. M. G. \& Matamala, Z. D. A. Aspectos biométricos del canal mandibular. Int. J. Morphol., 25(4):811-6, 2007.

Suazo, G. I. C.; Russo, P. P.; Zavando, M. D. A \& Smith, R. L. Sexual dimorphism in the foramen Magnum dimensions. Int. J. Morphol., 27(1):21-3, 2009b.

Suazo, G. I. C.; Zavando, M. D. A \& Smith, R. L. Sex determination using mastoid process measurements in Brazilian skulls. Int. J. Morphol., 26(4):941-4, 2008.

Suazo, G. I. C.; Zavando, M. D. A \& Smith, R. L. Sex Determination in mandibles in the first year of life by a quantitative approach. Int. J. Morphol., 27(1):113-6, 2009a.

Suazo, G. I. C.; Zavando, M. D. A \& Smith, R. L. Performance evaluation as a diagnostic test for traditional methods for forensic identification of sex. Int. J. Morphol., 27(2):381-6, 2009c.

Tatlisumak, E.; Ovali, G. Y.; Asirdizer, M.; Aslan, A.; Ozyurt, B.; Bayindir, P. \& Tarhan, S. CT study on morphometry of frontal sinus. Clin. Anat., 21(4):287-93, 2008.

Thompson, D. On Growth and Form. Cambridge, Cambridge University Press, 1917.

Toro, M. V. Tesis de Magíster en Ciencias Biológicas. Santiago, Universidad de Chile, 2010.

Wahl, C.; Li, T.; Choden, T. \& Howland, H. Morphometrics of corneal growth in chicks raised in constant light. $J$. Anat., 214(3):355-61, 2009.
Zavando, M. D. A; Suazo, G. I. C \& Smith, R. L. Sexual dimorphism determination in the lineal dimensions of skulls. Int. J. Morphol., 27(1):133-7, 2009a.

Zavando, M. D. A.; Suazo, G. I. C. \& Smith, R. L. ¿Es Posible la determinación de la afinidad racial a partir del análisis biométrico de cráneos humanos?. Int. J. Morphol., 27(3):643-8, 2009b.

Zelditch, M. L.; Swiderski, D. L.; Sheets, H. D. \& Fink, W. L. Geometric morphometrics for biologists: A primer. Elsevier Academic Press, 2004.

Zuccarelli, M. Comparative morphometric analysis of captive vs. wild African lion (Panthera leo) skulls. BIOS, 75(4):131-8, 2004.

Dirección para Correspondencia Dra. María Viviana Toro Ibacache Instituto de Ciencias Biomédicas Facultad de Medicina Universidad de Chile

Email: mtoro@med.uchile.cl

Recibido : 11-07-2010

Aceptado: 22-08-2010 